\title{
Separation of 2-propanol and water azeotropic system using ionic liquids as entrainers
}

Zhigang Zhang, Lin Zhang, Qinqin Zhang,* Dezhang Sun, Fenjin Pan, Shangwu Dai, and Wenxiu Li*

Liaoning Provincial Key Laboratory of Chemical Separation Technology, Shenyang University of Chemical Technology, Shenyang, 110142 , China.

\begin{abstract}
2-propanol and water form an azeotropic mixture of the minimum boiling point at constant pressure. In this work, three ionic liquids (ILs), namely 1-ethyl-3-methylimidazolium acetate ([EMIM][OAc]), 1-butyl-3-methylimidazolium acetate ([BMIM][OAc]) and 1-ethyl-3-methylimidazolium bromide ([EMIM] $[\mathrm{Br}])$, were used as entrainers to separate the azeotropic mixture by the method of extractive distillation. Isobaric vapor-liquid equilibrium (VLE) data for the ternary systems of 2-propanol + water + [EMIM][OAc], 2-propanol + water + [BMIM][OAc], and 2-propanol + water + [EMIM] $[\mathrm{Br}]$ were measured at $101.3 \mathrm{kPa}$. The results demonstrate that the relative volatility of 2-propanol to water is dramatically enhanced with the addition of ILs at areas of 2-propanol molar fraction higher than 0.2. As the amounts of ILs increase, the azeotropic point is pulled up and the azeotropy is even eliminated gradually. The separation effect (namely the effect of ILs on enhancement of the relative volatility) of the three ILs follows the order: $[\mathrm{EMIM}][\mathrm{OAc}]>[\mathrm{BMIM}][\mathrm{OAc}]>[\mathrm{EMIM}][\mathrm{Br}]$. Moreover, The experimental VLE data were well correlated with the nonrandom two-liquid model (NRTL).
\end{abstract}

Keywords: vapor-liquid equilibrium, ionic liquids, 2-propanol, water, NRTL model

* Corresponding Author: Wenxiu Li, Email address: wenxli@126.com, Fax: 86-24-89383736; 


\section{Introduction}

2-propanol is an important chemical product and raw material. Due to its excellent physico-chemical properties, 2-propanol finds its use in various areas, such as used as an intermediate or a solvent in pharmaceutical industry [1], applications in biofuels [2], and other engineering applications [3][4]. Generally, there are two commercial routes to produce 2-propanol, namely direct hydration and indirect hydration of propylene. [5] Therefore, it becomes necessary to separate 2-propanol from water. However, a separation problem occurs because 2-propanol and water form a minimum azeotrope at 2-propanol mass fraction of $87.4 \%$, and the azeotropic temperature is $353.45 \mathrm{~K}$ at $101.3 \mathrm{kPa}$. [6]

Due to the inefficiency of conventional distillation for azeotrope separation, it is indispensable to turn to special distillations, such as extractive distillation, pressure swing distillation, azeotropic distillation, salt adding distillation and so on. [7] In this study, the extractive distillation separation method draws our attention, for its high-efficiency separation capacity for binary azeotropes and other close boiling point mixtures. Extractive distillation, by adding a third solvent (namely entrainer) to the separated mixtures, achieves the goal of valid separation.[8] Hence, the selection of entrainers should be paid more attention. Traditional entrainers such as salts [9] and organic solvents [10] have been applied to separate the azeotropic mixture, but they have the disadvantages of corrosivity, pollution to the environment and difficulty in recycling.

In this work, ionic liquids (ILs) are proposed as entrainers because of their attractive advantages, such as non-volatility, non-flammability, high chemical and thermal stability, convenient recycling and tailorable structures.[11] During the past years, the isobaric or isothermal vapor-liquid equilibrium data for 2-propanol and water containing ionic liquids have been reported. Various ionic liquids have been investigated to test their abilities to break the azeotropy, including 1-ethyl-3-methylimidazole tetrafluoroborate [12], 1-butyl-3-methylimidazole tetrafluoroborate [6], 1-butyl-3-methylimidazole chloride [13] and 1-butyl-3-methylimidazole acetate [14]. The ILs [EMIM][OAc], [BMIM][OAc] have also been used to 
sepatate the mixture[15], but the experiment conclusion only can be made at the 2-propanol- rich region. In this study, the ILs [EMIM][OAc], [BMIM][OAc], [EMIM][Br] were utilized to separate the azeotropic mixture of 2-propanol and water. The VLE data expanded to water- rich region were measured at $101.3 \mathrm{kPa}$ and were correlated with the non-random two liquid (NRTL) model. By analyzing the results, we tried to find the potential entrainers for the system.

\section{Experimental section}

\subsection{Material}

2-propanol (mass fraction $\geq 99.7 \%$ ) used in this work was provided by Sinopharm Group without further purification. Water used in this work was ultrapure water produced by Water purification system (Direct-Q3UV). The ILs, [EMIM][OAc], [BMIM][OAc], and [EMIM][Br] were synthesized in our lab, and the mass purities of them were determined by Liquid Chromatography. The ILs were dried in a vacuum desiccator at $373 \mathrm{~K}$ for $24 \mathrm{~h}$ before experiment with the purpose of making the moisture content down to 0.003 (mass fraction) checked by Karl Fischer titration. Specifications of the chemicals utilized in this work are listed in Table 1.

\section{Table 1}

Overviews of the chemical samples

\begin{tabular}{lllll}
\hline Chemical name & Source & purity & Purification method & Analysis method \\
\hline 2-propanol & SinopharmGroup & 0.997 & None & $\mathrm{GC}^{\mathrm{a}}$ \\
{$[$ BMIM][OAc] } & Synthesized own & 0.990 & Vacuum desiccation & $\mathrm{LC}^{\mathrm{c}} \mathrm{KF}^{\mathrm{b}}$ \\
{$[$ EMIM][OAc] } & Synthesized own & 0.990 & Vacuum desiccation & $\mathrm{LC}^{\mathrm{c}} \mathrm{KF}^{\mathrm{b}}$ \\
{$[$ EMIM][Br] } & Synthesized own & 0.990 & Vacuum desiccation & $\mathrm{LC}^{\mathrm{c}} \mathrm{KF}^{\mathrm{b}}$ \\
\hline
\end{tabular}

\footnotetext{
${ }^{\mathrm{a}} \mathrm{GC}=$ gas chromatography.

${ }^{\mathrm{b}} \mathrm{KF}=$ Karl Fisher titration.

${ }^{\mathrm{c}} \mathrm{LC}=$ liquid chromatography.
}

\subsection{Apparatus and procedure}

In this wok, an all-glass dynamic recirculating still (NGW, Wertheim, Germany), which has been described in detail in the previous literature[16] [17], was used to conduct the experiment. The pressure of 
the apparatus was kept at $101.3 \mathrm{kPa}$ by controlling a gas buffer connected with the still. A manometer with an uncertainty of $0.1 \mathrm{kPa}$ was used to measure the pressure of the apparatus. A precise and calibrated thermometer with an uncertainty of $0.01 \mathrm{~K}$ was used to measure the temperature of the apparatus. The experiment was performed in the way that the binary mixture of water and defined mole fraction of ILs were added in batches into the other mixture of 2-propanol and the same mole fraction of IL continuously, with the purpose of changing the mole fraction of 2-propanol from zero to unity and keeping the mole fraction of IL in each series constant in the ternary system. Only when the temperature was constant for more than 30 min, was the VLE assumed.

Both the vapor and liquid phases were detected by a gas chromatograph (Model 7890A, Agilent Technologies) equipped with a $30 \mathrm{~m}, 0.32 \mathrm{~mm}$ i.d., and $0.25 \mu \mathrm{m}$ Agilent $19091 \mathrm{~J}-413$ capillary column and a TCD detector for which the carrier gas is $\mathrm{N}_{2}$. The operating conditions were as follows: the temperatures of oven, injector, and detector were 373,473 , and $473 \mathrm{~K}$, respectively. The samples were injected into the headspace sampler (G1888 Network. headspace Sampler Agilent Technologies) before being injected into the GC. Due to the non-volatility of ILs, only the peaks of 2-propanol and water were observed. A digital balance (CAV264C OHAUS America) with a standard uncertainty of $0.001 \mathrm{~g}$ was used to measure the content of ILs by weighting the sample before and after vaporizing the volatile component in a vacuum desiccator. And the standard uncertainty of the content of the component in the liquid and vapor phase is 0.001(mole fraction).

\section{Results and discussion}

\subsection{Experimental data}

With the purpose of verifying the reliability of the still used in this work, the binary VLE data of 2-propanol and water were determined at $101.3 \mathrm{kPa}$. The VLE data, listed in Table 2, were compared with the data calculated by the NRTL model and the data from literature [12]. As shown in Fig.1, the VLE data of 2-propanol and water system measured in this work agree well with the literature and the calculated data, 
indicating that the still used in the work is reliable.

Table 2

The isobaric VLE data for the binary system of 2-propanol (1) + Water (2) at $101.3 \mathrm{kPa}$.

\begin{tabular}{lll}
\hline$T(\mathrm{~K})$ & $x_{1}$ & $y_{1}$ \\
\hline 365.42 & 0.023 & 0.307 \\
358.73 & 0.049 & 0.433 \\
356.22 & 0.078 & 0.502 \\
354.91 & 0.126 & 0.522 \\
354.34 & 0.248 & 0.544 \\
354.15 & 0.373 & 0.554 \\
353.82 & 0.448 & 0.574 \\
353.43 & 0.562 & 0.624 \\
353.36 & 0.625 & 0.645 \\
353.32 & 0.675 & 0.674 \\
353.45 & 0.747 & 0.710 \\
353.76 & 0.825 & 0.790 \\
353.88 & 0.865 & 0.831 \\
354.65 & 0.948 & 0.928 \\
\hline Stand & &
\end{tabular}

Standard uncertainty $u\left(x_{1}\right)=u\left(y_{1}\right)= \pm 0.001, u(\mathrm{~T})= \pm 0.01 \mathrm{~K}$

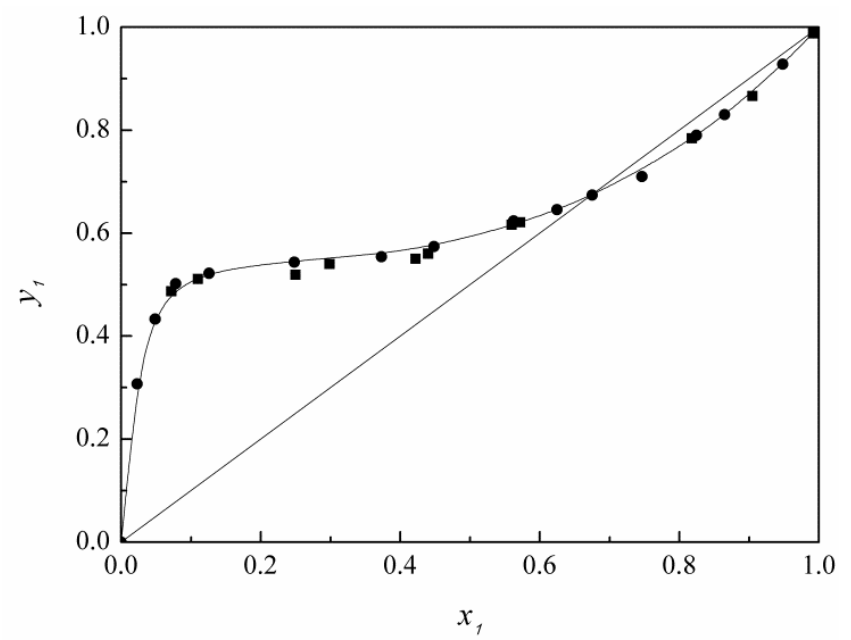

Fig. 1 Comparison of isobaric VLE data for the binary system of 2-propanol (1) + water (2) at $101.3 \mathrm{kPa}$ : , this work; $\mathbf{\square}$, ref [12]; solid line, the correlated data using the NRTL model.

As mentioned above, the relative volatility $\alpha_{12}$ is considered as an important indicative quantity to evaluate the separation effect of an entrainer, and is defined as

$$
\alpha_{12}=\frac{y_{1} / x_{1}}{y_{2} / x_{2}}
$$

where $x_{1}\left(x_{2}\right)$ and $y_{1}\left(y_{2}\right)$ are the mole fractions of component 1(2) in the liquid phase and vapor phase, respectively.

Since the experimental data were measured at $101.3 \mathrm{kPa}$, the assumption of the ideal vapor phase could 
be made. After neglecting the fugacity coefficients and the Poynting correction, the VLE equation can be simplified as:

$$
y_{i} P=x_{i} \gamma_{i} P_{i}^{s}
$$

where $y_{\mathrm{i}}$ and $x_{\mathrm{i}}$ represent the mole fraction of components $\mathrm{i}$ in the vapor phase and the liquid phase, respectively, $P$ is the total pressure of $101.3 \mathrm{kPa}, \gamma_{\mathrm{i}}$ is the activity coefficient of component $\mathrm{i}$ in the liquid phase, $P_{\mathrm{i}}^{\mathrm{s}}$ is the saturated vapor pressure of component $\mathrm{i}$ at equilibrium temperature which can be calculated using the Antoine equation:

$$
\log P_{i}^{s}=A_{\mathrm{i}}-\frac{B_{\mathrm{i}}}{t+C_{\mathrm{i}}}
$$

where $P_{\mathrm{i}}^{\mathrm{s}}$ is the saturated vapor pressure of component $\mathrm{i}$ at equilibrium temperature in $\mathrm{mmHg}, t$ is the equilibrium temperature in ${ }^{\circ} \mathrm{C}$, and $A_{\mathrm{i}}, B_{\mathrm{i}}$, and $C_{\mathrm{i}}$ are the Antoine parameters of $\mathrm{i}$. The Antoine parameters of 2-propanol [18]and water from the Aspen Plus are listed in Table 3.

\section{Table 3}

Antoine parameters for 2-propanol and water.

\begin{tabular}{llllll}
\hline \multirow{2}{*}{ Component } & \multicolumn{2}{l}{ Antoine parameters } & \multicolumn{3}{c}{ Temperature range } \\
\cline { 2 - 6 } & $A_{\mathrm{i}}$ & $B_{\mathrm{i}}$ & $C_{\mathrm{i}}$ & $\mathrm{Min} /\left({ }^{\circ} \mathrm{C}\right)$ & $\mathrm{Max} /\left({ }^{\circ} \mathrm{C}\right)$ \\
2-propanol & 8.1182 & 1580.92 & 219.62 & 0 & 133 \\
Water & 7.96681 & 1668.21 & 228 & 60 & 150 \\
\hline
\end{tabular}

The VLE data for the 2-propanol (1) + water (2) + [EMIM][OAc] (3), 2-propanol (1)+ water (2) + $[\mathrm{BMIM}][\mathrm{OAc}](3), 2-\operatorname{propanol}(1)+$ water $(2)+[\mathrm{EMIM}][\mathrm{Br}](3)$ ternary systems measured at $101.3 \mathrm{kPa}$ are listed in Tables 4-6, where $x_{3}$ represents the mole fraction of ILs in the liquid phase, $x_{1}$ represents the mole fraction of 2-propanol in the liquid phase containing ILs, $x_{1}$ ' represents the mole fraction of 2-propanol in the liquid phase on an IL-free basis, $y_{1}$ is the mole fraction of 2-propanol in the vapor phase, $T$ is the equilibrium temperature, $\alpha_{12}$ is the relative volatility of 2-propanol to water calculated by the equation (1). The VLE data were measured by keeping the mole fractions of ILs nearly constant at $0.1,0.2$, and 0.3 , respectively. 
Table 4

Isobaric VLE data for ternary system of 2-propanol (1) + water (2) + [EMIM][OAc] (3) at $101.3 \mathrm{kPa}$.

\begin{tabular}{|c|c|c|c|c|c|c|c|c|c|c|c|}
\hline$T(\mathrm{~K})$ & $x_{3}$ & $x_{1}$ & $x_{1}{ }^{\prime}$ & $y_{1}$ & $\alpha_{12}$ & $T(\mathrm{~K})$ & $x_{3}$ & $x_{1}$ & $x_{1}^{\prime}$ & $y_{1}$ & $\alpha_{12}$ \\
\hline \multicolumn{12}{|l|}{$x_{3} \approx 0.1$} \\
\hline 357.08 & 0.124 & 0.876 & 1.000 & 1.000 & & 358.67 & 0.124 & 0.366 & 0.418 & 0.758 & 4.361 \\
\hline 356.67 & 0.121 & 0.858 & 0.976 & 0.977 & 1.044 & 360.08 & 0.127 & 0.340 & 0.389 & 0.745 & 4.589 \\
\hline 356.37 & 0.123 & 0.796 & 0.908 & 0.937 & 1.507 & 362.38 & 0.126 & 0.263 & 0.301 & 0.686 & 5.073 \\
\hline 356.04 & 0.127 & 0.726 & 0.832 & 0.907 & 1.969 & 364.16 & 0.126 & 0.200 & 0.229 & 0.619 & 5.470 \\
\hline 355.66 & 0.124 & 0.627 & 0.716 & 0.866 & 2.563 & 366.56 & 0.120 & 0.136 & 0.154 & 0.516 & 5.857 \\
\hline 355.84 & 0.127 & 0.569 & 0.652 & 0.849 & 3.000 & 369.95 & 0.109 & 0.046 & 0.052 & 0.257 & 6.306 \\
\hline 356.14 & 0.126 & 0.485 & 0.555 & 0.816 & 3.556 & 373.55 & 0.112 & 0.000 & 0.000 & 0.000 & \\
\hline 357.17 & 0.124 & 0.427 & 0.487 & 0.789 & 3.939 & & & & & & \\
\hline \multicolumn{12}{|l|}{$x_{3} \approx 0.2$} \\
\hline 360.26 & 0.202 & 0.798 & 1.000 & 1.000 & & 363.35 & 0.196 & 0.324 & 0.403 & 0.785 & 5.409 \\
\hline 359.03 & 0.207 & 0.780 & 0.984 & 0.992 & 2.016 & 365.54 & 0.192 & 0.218 & 0.270 & 0.688 & 5.962 \\
\hline 358.74 & 0.194 & 0.728 & 0.903 & 0.962 & 2.719 & 367.51 & 0.192 & 0.160 & 0.198 & 0.609 & 6.309 \\
\hline 358.58 & 0.200 & 0.666 & 0.832 & 0.941 & 3.220 & 370.43 & 0.192 & 0.128 & 0.158 & 0.550 & 6.513 \\
\hline 358.77 & 0.200 & 0.572 & 0.715 & 0.906 & 3.842 & 372.73 & 0.191 & 0.080 & 0.099 & 0.424 & 6.700 \\
\hline 359.97 & 0.196 & 0.478 & 0.594 & 0.866 & 4.417 & 375.53 & 0.193 & 0.043 & 0.053 & 0.282 & 7.018 \\
\hline 361.53 & 0.194 & 0.392 & 0.486 & 0.825 & 4.986 & 376.28 & 0.213 & 0.000 & 0.000 & 0.000 & \\
\hline \multicolumn{12}{|l|}{$x_{3} \approx 0.3$} \\
\hline 359.77 & 0.316 & 0.684 & 1.000 & 1.000 & & 366.65 & 0.298 & 0.298 & 0.424 & 0.837 & 6.976 \\
\hline 359.73 & 0.286 & 0.667 & 0.934 & 0.985 & 4.640 & 369.37 & 0.297 & 0.225 & 0.320 & 0.777 & 7.404 \\
\hline 359.67 & 0.288 & 0.634 & 0.891 & 0.976 & 4.975 & 371.14 & 0.296 & 0.185 & 0.263 & 0.729 & 7.538 \\
\hline 359.75 & 0.291 & 0.581 & 0.820 & 0.960 & 5.268 & 373.04 & 0.299 & 0.153 & 0.219 & 0.683 & 7.684 \\
\hline 360.02 & 0.289 & 0.524 & 0.738 & 0.941 & 5.662 & 375.96 & 0.300 & 0.090 & 0.129 & 0.540 & 7.926 \\
\hline 360.55 & 0.292 & 0.481 & 0.679 & 0.927 & 6.003 & 377.16 & 0.300 & 0.060 & 0.086 & 0.429 & 7.985 \\
\hline 361.32 & 0.296 & 0.439 & 0.624 & 0.913 & 6.323 & 379.25 & 0.299 & 0.000 & 0.000 & 0.000 & \\
\hline 362.82 & 0.296 & 0.381 & 0.541 & 0.887 & 6.660 & & & & & & \\
\hline
\end{tabular}

Standard uncertainty $u\left(x_{1}{ }^{\prime}\right)=u\left(x_{1}\right)=u\left(y_{1}\right)= \pm 0.001 u(T)= \pm 0.01 \mathrm{~K}$. 
Table 5

Isobaric VLE data for ternary system of 2-propanol (1) + water (2) + [BMIM][OAc] (3) at $101.3 \mathrm{kPa}$.

\begin{tabular}{|c|c|c|c|c|c|c|c|c|c|c|c|}
\hline$T(\mathrm{~K})$ & $x_{3}$ & $x_{1}$ & $x_{1}^{\prime}$ & $y_{1}$ & $\alpha_{12}$ & $T(\mathrm{~K})$ & $x_{3}$ & $x_{1}$ & $x_{1}{ }^{\prime}$ & $y_{1}$ & $\alpha_{12}$ \\
\hline \multicolumn{12}{|l|}{$x_{3} \approx 0.1$} \\
\hline 356.84 & 0.102 & 0.898 & 1.000 & 1.000 & & 358.01 & 0.120 & 0.289 & 0.328 & 0.675 & 4.259 \\
\hline 355.68 & 0.112 & 0.741 & 0.834 & 0.875 & 1.398 & 358.82 & 0.114 & 0.253 & 0.286 & 0.642 & 4.467 \\
\hline 355.64 & 0.115 & 0.668 & 0.755 & 0.846 & 1.777 & 359.82 & 0.116 & 0.218 & 0.247 & 0.606 & 4.691 \\
\hline 355.66 & 0.112 & 0.580 & 0.653 & 0.814 & 2.329 & 360.94 & 0.113 & 0.162 & 0.183 & 0.526 & 4.956 \\
\hline 355.86 & 0.118 & 0.484 & 0.549 & 0.781 & 2.935 & 363.90 & 0.114 & 0.074 & 0.084 & 0.331 & 5.398 \\
\hline 356.18 & 0.119 & 0.426 & 0.484 & 0.756 & 3.305 & 365.91 & 0.115 & 0.037 & 0.042 & 0.200 & 5.706 \\
\hline 356.64 & 0.118 & 0.385 & 0.436 & 0.734 & 3.569 & 373.15 & 0.120 & 0.000 & 0.000 & 0.000 & \\
\hline 357.24 & 0.117 & 0.325 & 0.368 & 0.700 & 4.007 & & & & & & \\
\hline \multicolumn{12}{|l|}{$x_{3} \approx 0.2$} \\
\hline 357.76 & 0.236 & 0.764 & 1.000 & 1.000 & & 362.24 & 0.234 & 0.230 & 0.300 & 0.665 & 4.632 \\
\hline 357.55 & 0.232 & 0.686 & 0.893 & 0.949 & 2.230 & 363.13 & 0.230 & 0.191 & 0.248 & 0.613 & 4.803 \\
\hline 357.25 & 0.234 & 0.617 & 0.805 & 0.916 & 2.642 & 365.12 & 0.230 & 0.174 & 0.226 & 0.586 & 4.846 \\
\hline 357.31 & 0.235 & 0.557 & 0.728 & 0.888 & 2.962 & 367.14 & 0.231 & 0.141 & 0.183 & 0.529 & 5.014 \\
\hline 357.34 & 0.238 & 0.471 & 0.618 & 0.847 & 3.422 & 368.57 & 0.235 & 0.097 & 0.127 & 0.428 & 5.143 \\
\hline 357.55 & 0.231 & 0.439 & 0.571 & 0.827 & 3.591 & 372.63 & 0.234 & 0.056 & 0.073 & 0.295 & 5.314 \\
\hline 358.06 & 0.232 & 0.379 & 0.493 & 0.792 & 3.916 & 374.35 & 0.234 & 0.030 & 0.039 & 0.182 & 5.482 \\
\hline 359.12 & 0.230 & 0.333 & 0.432 & 0.759 & 4.141 & 377.25 & 0.233 & 0.000 & 0.000 & 0.000 & \\
\hline 361.03 & 0.232 & 0.276 & 0.359 & 0.712 & 4.414 & & & & & & \\
\hline \multicolumn{12}{|l|}{$x_{3} \approx 0.3$} \\
\hline 356.84 & 0.341 & 0.659 & 1.000 & 1.000 & & 356.64 & 0.342 & 0.315 & 0.479 & 0.807 & 4.548 \\
\hline 355.94 & 0.343 & 0.608 & 0.925 & 0.976 & 3.300 & 357.24 & 0.341 & 0.273 & 0.414 & 0.768 & 4.686 \\
\hline 355.68 & 0.340 & 0.567 & 0.859 & 0.955 & 3.484 & 358.01 & 0.343 & 0.204 & 0.310 & 0.687 & 4.880 \\
\hline 355.64 & 0.338 & 0.506 & 0.766 & 0.925 & 3.768 & 358.82 & 0.343 & 0.174 & 0.265 & 0.643 & 5.000 \\
\hline 355.66 & 0.341 & 0.468 & 0.710 & 0.907 & 3.983 & 359.82 & 0.342 & 0.126 & 0.191 & 0.545 & 5.073 \\
\hline 355.86 & 0.338 & 0.435 & 0.657 & 0.887 & 4.098 & 363.00 & 0.339 & 0.087 & 0.132 & 0.439 & 5.146 \\
\hline 356.18 & 0.341 & 0.374 & 0.568 & 0.851 & 4.344 & 364.64 & 0.336 & 0.000 & 0.000 & 0.000 & \\
\hline
\end{tabular}

Standard uncertainty $u\left(x_{1}^{\prime}\right)=u\left(x_{1}\right)=u\left(y_{1}\right)= \pm 0.001, u(T)= \pm 0.01 \mathrm{~K}$. 
Table 6

Isobaric VLE data for ternary system of 2-propanol (1) + water (2) + [EMIM][Br] (3) at $101.3 \mathrm{kPa}$.

\begin{tabular}{|c|c|c|c|c|c|c|c|c|c|c|c|}
\hline$T(\mathrm{~K})$ & $x_{3}$ & $x_{1}$ & $x_{1}^{\prime}$ & $y_{1}$ & $\alpha_{12}$ & $T(\mathrm{~K})$ & $x_{3}$ & $x_{1}$ & $x_{1}^{\prime}$ & $y_{1}$ & $\alpha_{12}$ \\
\hline \multicolumn{12}{|l|}{$x_{3} \approx 0.1$} \\
\hline 355.24 & 0.116 & 0.884 & 1.000 & 1.000 & & 357.10 & 0.116 & 0.364 & 0.412 & 0.724 & 3.744 \\
\hline 354.95 & 0.113 & 0.854 & 0.963 & 0.938 & 0.581 & 360.00 & 0.118 & 0.288 & 0.326 & 0.681 & 4.414 \\
\hline 354.37 & 0.117 & 0.784 & 0.888 & 0.876 & 0.891 & 362.10 & 0.114 & 0.208 & 0.235 & 0.608 & 5.049 \\
\hline 354.10 & 0.111 & 0.736 & 0.828 & 0.842 & 1.107 & 364.47 & 0.115 & 0.159 & 0.180 & 0.544 & 5.435 \\
\hline 353.74 & 0.115 & 0.654 & 0.739 & 0.817 & 1.577 & 368.30 & 0.112 & 0.101 & 0.114 & 0.432 & 5.911 \\
\hline 353.95 & 0.110 & 0.614 & 0.690 & 0.804 & 1.843 & 369.05 & 0.104 & 0.076 & 0.085 & 0.361 & 6.081 \\
\hline 353.97 & 0.110 & 0.562 & 0.631 & 0.790 & 2.200 & 369.45 & 0.114 & 0.046 & 0.052 & 0.258 & 6.339 \\
\hline 354.70 & 0.116 & 0.513 & 0.580 & 0.780 & 2.567 & 371.45 & 0.115 & 0.020 & 0.023 & 0.133 & 6.516 \\
\hline 355.56 & 0.112 & 0.454 & 0.511 & 0.759 & 3.014 & 372.22 & 0.116 & 0.000 & 0.000 & 0.000 & \\
\hline \multicolumn{12}{|l|}{$x_{3} \approx 0.2$} \\
\hline 357.21 & 0.199 & 0.801 & 1.000 & 1.000 & & 356.45 & 0.206 & 0.313 & 0.394 & 0.748 & 4.565 \\
\hline 356.10 & 0.198 & 0.746 & 0.930 & 0.939 & 1.159 & 357.86 & 0.206 & 0.203 & 0.256 & 0.656 & 5.542 \\
\hline 355.85 & 0.203 & 0.686 & 0.861 & 0.911 & 1.652 & 360.04 & 0.206 & 0.150 & 0.189 & 0.583 & 5.999 \\
\hline 355.83 & 0.208 & 0.645 & 0.814 & 0.893 & 1.907 & 362.22 & 0.205 & 0.088 & 0.111 & 0.446 & 6.448 \\
\hline 355.57 & 0.209 & 0.570 & 0.721 & 0.862 & 2.417 & 364.25 & 0.209 & 0.055 & 0.070 & 0.338 & 6.783 \\
\hline 355.50 & 0.201 & 0.496 & 0.621 & 0.829 & 2.959 & 367.15 & 0.207 & 0.032 & 0.040 & 0.226 & 7.008 \\
\hline 355.36 & 0.206 & 0.416 & 0.524 & 0.800 & 3.634 & 368.24 & 0.205 & 0.000 & 0.000 & 0.000 & \\
\hline 355.58 & 0.204 & 0.370 & 0.465 & 0.778 & 4.032 & & & & & & \\
\hline \multicolumn{12}{|l|}{$x_{3} \approx 0.3$} \\
\hline 356.82 & 0.308 & 0.692 & 1.000 & 1.000 & & 360.00 & 0.303 & 0.224 & 0.321 & 0.743 & 6.115 \\
\hline 356.43 & 0.301 & 0.659 & 0.943 & 0.980 & 2.962 & 361.70 & 0.307 & 0.180 & 0.260 & 0.695 & 6.485 \\
\hline 356.76 & 0.302 & 0.610 & 0.874 & 0.955 & 3.059 & 363.74 & 0.303 & 0.132 & 0.189 & 0.616 & 6.883 \\
\hline 357.05 & 0.302 & 0.538 & 0.771 & 0.919 & 3.370 & 365.57 & 0.302 & 0.097 & 0.139 & 0.537 & 7.184 \\
\hline 357.10 & 0.310 & 0.455 & 0.659 & 0.888 & 4.103 & 367.56 & 0.304 & 0.051 & 0.073 & 0.374 & 7.587 \\
\hline 357.77 & 0.304 & 0.370 & 0.532 & 0.844 & 4.759 & 371.26 & 0.304 & 0.013 & 0.018 & 0.126 & 7.856 \\
\hline 358.57 & 0.304 & 0.284 & 0.408 & 0.793 & 5.558 & 372.55 & 0.304 & 0.000 & 0.000 & 0.000 & \\
\hline
\end{tabular}

Standard uncertainty $u\left(x_{1}^{\prime}\right)=u\left(x_{1}\right)=u\left(y_{1}\right)= \pm 0.001, u(T)= \pm 0.01 \mathrm{~K}$.

3.2. Calculation of the Phase Equilibrium

The NRTL model proposed by Renon and Prausnitz [19] is frequently used for the experimental VLE data regression of the IL-containing system because it often fits the experimental data well. In this work, the NRTL model is also used to correlate the experimental VLE data. To calculate the ternary VLE data, nine parameters of the NRTL model should be determined, including six binary energy parameters and three non-randomness factors. The parameters for 2-propanol and water were obtained from literature [20]. The other six parameters were regressed by the NRTL model and experimental data and are listed in Table 7. 


\section{Table 7}

Nonrandom factors and binary energy parameters for the NRTL model.

\begin{tabular}{lllll} 
Component $i$ & Component $j$ & $\alpha_{\mathrm{i}, \mathrm{j}}$ & \multicolumn{1}{c}{$\Delta g_{i, j}$} & \multicolumn{1}{c}{$\Delta g_{j, i}$} \\
& & & $J / \mathrm{mol}$ & $77.49^{\mathrm{a}}$ \\
\hline 2-propanol & Water & $0.300^{\mathrm{a}}$ & $6900.81^{\mathrm{a}}$ & -985.6 \\
2-propanol & {$[$ EMIM][OAc] } & 0.200 & -5680.2 & -7000.2 \\
Water & {$[$ EMIM][OAc] } & 0.26 & -6580.2 & -1185.4 \\
2 -propanol & {$[\mathrm{BMIM}][\mathrm{OAc}]$} & 0.300 & -6255.6 & -6344.8 \\
Water & {$[\mathrm{BMIM}][\mathrm{OAc}]$} & 0.200 & -5442.5 & 123.23 \\
2-propanol & {$[$ EMIM][Br] } & 0.018 & -2458.27 & 20380.41 \\
Water & {$[$ EMIM][Br] } & 0.455 & -8990.65 & \\
\hline
\end{tabular}

${ }^{\mathrm{a}}$ From ref. [20], $\Delta g_{\mathrm{i}, \mathrm{j}}=g_{\mathrm{i}, \mathrm{j}}-g_{\mathrm{i}, \mathrm{i}}, \tau_{\mathrm{ij}}=\left(g_{\mathrm{i}, \mathrm{j}}-g_{\mathrm{i}, \mathrm{i}}\right) / \mathrm{R} T$

Figs. 2-4 shows the isobaric $y_{1}-x_{1}{ }^{\prime}$ diagram for the 2-propanol (1) + water (2) + [EMIM][OAc] (3), [BMIM][OAc] (3) or [EMIM][Br] (3) ternary systems at $101.3 \mathrm{kPa}$ on an IL-free basis. As can be seen, at areas of higher 2-propanol content, the addition of a small quantity of ILs can significantly increase the content of 2-propanol in the vapor phase, producing a salting-out effect. Moreover, the azeotropic point of 2-propanol and water shifts towards areas of higher 2-propanol contents, and can be totally eliminated with increasing the amount of ILs to a specific value. For [EMIM][OAc], the azeotrope is broken when its mole fraction is larger than 0.1 , while the amount of [BMIM][OAc] and [EMIM][Br] needed is greater. According to the NRTL model, the minimum mole fractions of [EMIM][OAc], [BMIM][OAc] and [EMIM][Br] needed to break the azeotrope are $0.1184,0.1503,0.1989$, respectively. 


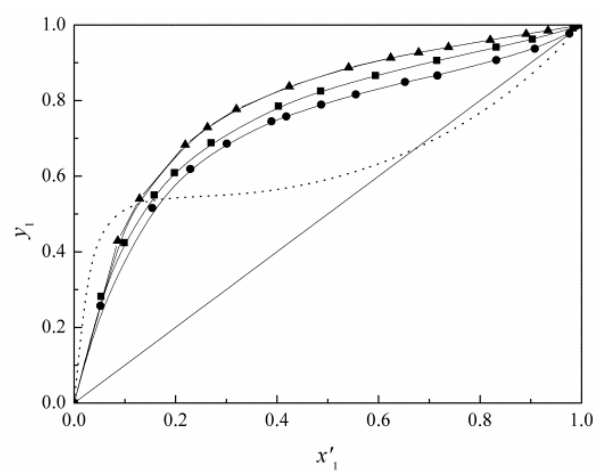

Fig. 2. Isobaric $y_{1}-x_{1}{ }^{\prime}$ diagram for the 2-propanol (1) + water (2) + [EMIM][OAc] (3) ternary system at $101.3 \mathrm{kPa}$ : dotted line, IL-free system; $\bullet, x_{3}=0.1 ; \mathbf{\square}, x_{3}=0.2 ; \mathbf{\Delta}, x_{3}=0.3$; solid line, correlated using NRTL model.

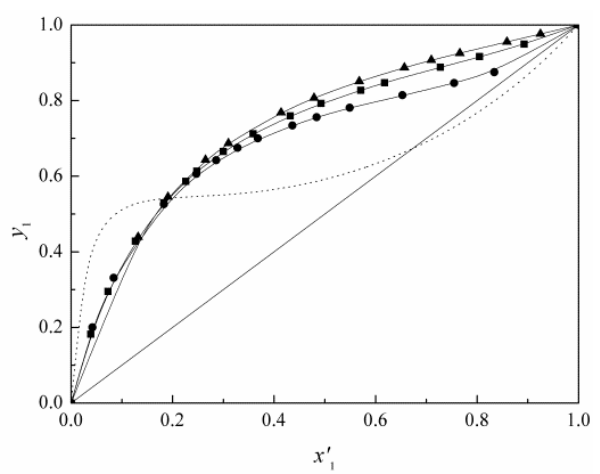

Fig. 3. Isobaric $y_{1}-x_{1}{ }^{\prime}$ diagram for the 2-propanol (1) + water (2) + [BMIM][OAc] (3) ternary system at $101.3 \mathrm{kPa}$ : dotted line, IL-free system; $\bullet, x_{3}=0.1 ; \boldsymbol{\square}, x_{3}=0.2 ; \boldsymbol{\Delta}, x_{3}=0.3$; solid line, correlated using NRTL model.

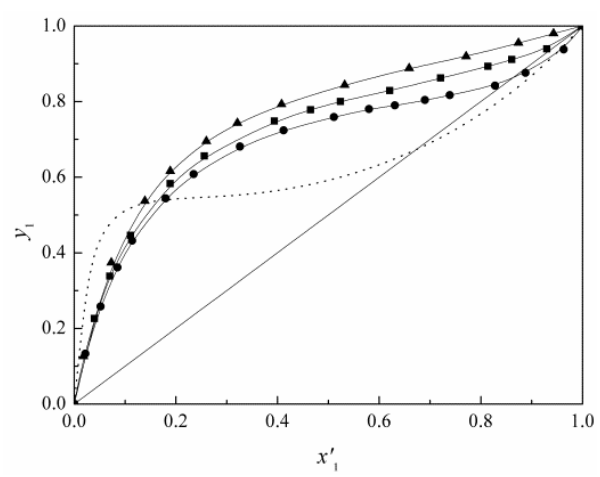

Fig. 4. Isobaric $y_{1}-x_{1}{ }^{\prime}$ diagram for the 2-propanol (1) + water (2) + [EMIM][Br] (3) ternary system at $101.3 \mathrm{kPa}$ : dotted line, IL-free system; $\bullet, x_{3}=0.1 ; \mathbf{\square}, x_{3}=0.2 ; \mathbf{\Delta}, x_{3}=0.3$; solid line, correlated using NRTL model.

The relative activities $\left(\alpha_{12}\right)$ of 2-propanol to water containing different ILs are illustrated in Figs. 5-7. It is obvious that the addition of small amounts of ILs significantly enhance the relative volatility of 2-propanol to water at areas of higher 2-propanol contents. Moreover, the more the addtion of ILs, the larger the relative volatilities of 2-propanol to water, consequently the easier the separation. At areas of higher 2-propanol contents, with the same amount of ILs, the sequence of the value of $\alpha_{12}$ varing with different ILs is: $[\mathrm{EMIM}][\mathrm{OAc}]>[\mathrm{BMIM}][\mathrm{OAc}]>[\mathrm{EMIM}][\mathrm{Br}]$. At areas of higher water contents, ILs show complex effects on the mixture of 2-propanol and water. In general, among the three ILs, [EMIM][OAc] produces the most obvious salting-out effect, followed by [BMIM][OAc] and [EMIM][Br], which is consistent with the result of literature[15]. 


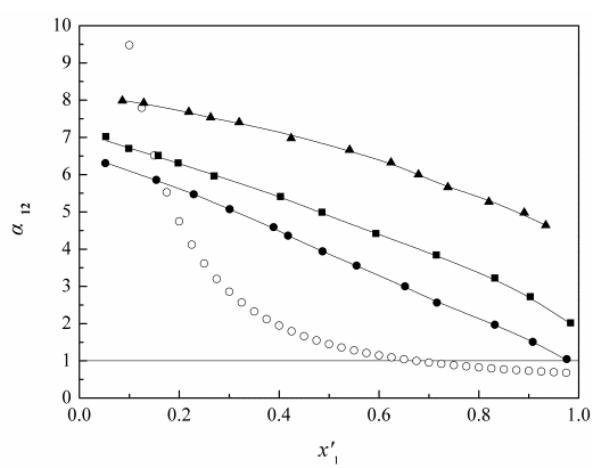

Fig. 5. Relative activity $a_{12}$ of 2-propanol (1) to water (2) at different mole fraction of [EMIM][OAc] at $101.3 \mathrm{kPa}: \circ, x_{3}=0 ; \bullet, x_{3}=0.1 ; \boldsymbol{\square}, x_{3}=$ $0.2 ; \boldsymbol{\Lambda}, x_{3}=0.3$; solid lines, correlated using NRTL model.

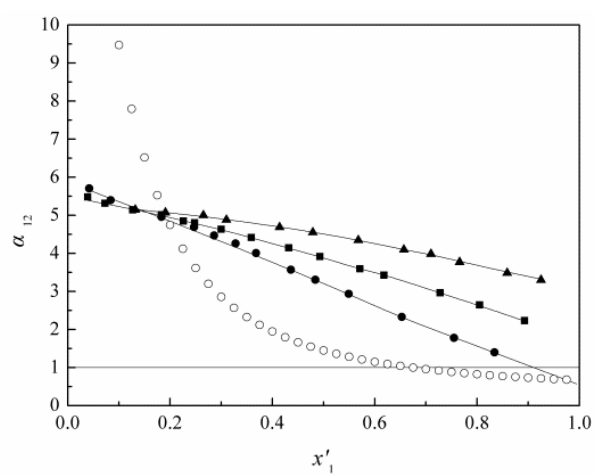

Fig. 6. Relative activity $a_{12}$ of 2-propanol (1) to water (2) at different mole fraction of [BMIM][OAc] at $101.3 \mathrm{kPa}: \circ, x_{3}=0 ; \bullet, x_{3}=0.1 ; \mathbf{\square}, x_{3}=$ $0.2 ; \boldsymbol{\Delta}, x_{3}=0.3$; solid lines, correlated using NRTL model.

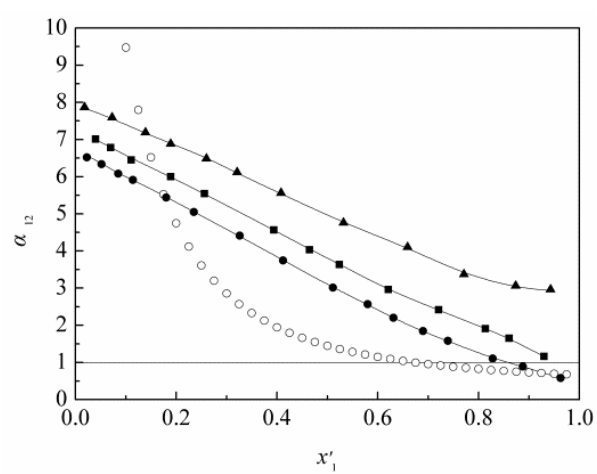

Fig. 7. Relative activity $a_{12}$ of 2-propanol (1) to water (2) at different mole fraction of [EMIM][Br] at 101.3 $\mathrm{kPa}: \circ, x_{3}=0 ; \bullet, x_{3}=0.1 ; \boldsymbol{\square}, x_{3}=0.2 ;$ $\boldsymbol{\Delta}, x_{3}=0.3 ;$ solid lines, correlated using NRTL model.

The effect of [EMIM][OAc], [BMIM][OAc] and [EMIM][Br] on the VLE behavior of the 2-propanol and water system might be attributed to the different molecular interactions between ILs and solvents. Molecular interactions are mainly electrostatic interaction, hydrogen bonding and Vander Waals forces.[21] In this work, the hydrogen bond is discussed.[22] As water is a stronger hydrogen bond donator than 2-propanol and the polarity of water is greater than that of 2-propanol, the interaction of ILs with water is stronger than that with 2-propanol, making 2-propanol more volatile compared to the IL-free system [24]. While at areas of higher water contents, in spite of the stronger molecular interaction between water molecules and ILs, more and more 2-propanol molecules are also interacted, thus making relative volatility of 2-propanol to water decreasing (at $x_{1}{ }^{\prime}$ below 0.2 ). To our knowledge, the anions of ILs play a key role in the molecular interactions of ILs with water.[23] Because the acetate ion can form a stronger hydrogen bond with water than bromide ion, the relative volatility of 2-propanol to water containing ILs with acetate ion is superior to that containing ILs with bromide ion. And for [EMIM][OAc] and [BMIM][OAc], the difference lies in the cation of ILs. As we all know, the weaker the polarity of the cation, the weaker the hydrogen bond to be formed. [21] [25] As the $[\mathrm{EMIM}]^{+}$cation has a shorter alkyl chain than $[\mathrm{BMIM}]^{+}$cation, the polarity of $[\mathrm{EMIM}][\mathrm{OAc}]$ is stronger than that of $[\mathrm{BMIM}][\mathrm{OAc}]$, thus $[\mathrm{EMIM}][\mathrm{OAc}]$ can form a stronger hydrogen 
bond with water, making 2-propanol more volatile compare to [BMIM][OAc]. So [EMIM][OAc] has a better separation effect than [BMIM][OAc]. As a result, [EMIM][OAc] could be expected to have a potential application for the separation of 2-propanol and water.

\section{Conclusions}

The VLE data of the 2-propanol (1) + water (2) + [EMIM][OAc] (3), 2-propanol (1) + water (2) + [BMIM][OAc] (3), 2-propanol (1) + water (2) + [EMIM][Br] (3) ternary systems were measured at 101.3 $\mathrm{kPa}$. All the three ILs produced a salting-out effect on 2-propanol at areas of higher 2-propanol content, enhancing the relative volatility of 2-propanol to water, and could eventually eliminate the azeotropic point with the increase of the amounts of ILs. [EMIM][OAc] has a better separation effect than the other two ILs, thus can be selected as a potential entrainer for the separation of the azeotropic mixture. In addition, the NRTL model can be applied to the IL-containing systems with good accuracy.

\section{Acknowledgement}

This work is financially supported by National Science Foundation of China (Project No. 21076126), National Science Foundation of China (Project No. 21576166), and Liaoning Province Science Foundation of China (Project No. 2014020140).

List of symbols

$\Delta g_{\mathrm{ij}}$

$x_{\mathrm{i}}$

$y_{\mathrm{i}}$

$T$

$P$

$P_{i}^{\mathrm{s}}$

Greek letters

$\alpha_{12}$

$\alpha_{\mathrm{ij}}$

$\gamma_{i}$ binary energy parameter of NRTL model

mole fraction of solvent $i$ in the liquid phase

mole fraction of solvent $i$ in the vapor phase

equilibrium temperature

total pressure in the equilibrium system

Saturated vapor pressure of component $i$

separation factor of component 1 to component 2

non-randomness parameter of NRTL model

activity coefficient of component $i$ 


\section{References}

[1] A. A. Sizikov, A. Yu. Manakov, Double gas hydrate of isopropanol and methane. Fluid Phase Equilib. 371 (2014) 75-81.

[2] B. Likozar, J. Levec, Transesterification of canola, palm, peanut, soybean and sunflower oil with methanol, ethanol, isopropanol, butanol and tert-butanol to biodiesel: Modelling of chemical equilibrium, reaction kinetics and mass transfer based on fatty acid composition. Appl. Energy. 123 (2014) 108-120.

[3] X-Y. Wang, X-D. Li, G. Tang, L-X. Zhao, W-J. Zhang, T-G. Jiu, J-F. Fang, Improving efficiency of planar hybrid $\mathrm{CH}_{3} \mathrm{NH}_{3} \mathrm{PbI}_{3-\mathrm{x}} \mathrm{Cl}_{\mathrm{x}}$ perovskite solar cells by isopropanol solvent treatment. Org. Electron. 24 (2015) 205-211.

[4] B. Burrichter, C. Pasel, M. Luckas, D. Bathen, Parameter study on the adsorptive drying of isopropanol in a fixed bed adsorber. Sep. Purif. Technol. 132 (2014) 736-743.

[5] J. I. Kroschwitz, Kirk - Othmer Encyclopedia of Chemical Technology, 4th Edition. John Wiley \& Sons, New York, USA 20 (1991) 216-240.

[6] Q-S. Li, J-G. Zhang, Z-G. Lei, J-Q. Zhu, B-H. Wang and X-Q. Huang, Isobaric Vapor-Liquid Equilibrium for (Propan-2-ol + Water + 1-Butyl-3-methylimidazolium Tetrafluoroborate). J. Chem. Eng. Data 54 (2009) 2785-2788.

[7] Z. Lei, B. Chen, Z. Ding, Special Distillation Processes .Elsevier: Amsterdam, 2005.

[8] M. I. Zaretskii, V. V. Rusak, and E. M. Chartov, Extractive distillation in chemical technology: a review, Coke and chemistry 53 (2010) 36-40.

[9] J. Bao. Y. M. Zhang, X. Jin, Vapor-Liquid Equilibria for the Isopropanol - Water - Mixed Solvent Containing Salt Systems. J. Chem. Eng. Chin. UniV. 19 (2005) 258-262.

[10] A. Font, J. Asensi, F. Ruiz, and Vicente Gomis, Isobaric Vapor - Liquid and Vapor - Liquid - Liquid Equilibria Data for the System Water + Isopropanol + Isooctane. J. Chem. Eng. Data. 49 (2004) 765-767.

[11]Alfredo Ortiz, Lara María Galán, Daniel Gorri, André B. de Haan and Inmaculada Ortiz, Reactive Ionic 
Liquid Media for the Separation of Propylene / Propane Gaseous Mixtures. Ind. Eng. Chem. Res. 49 (2010) 7227-7233.

[12] Q-S. Li, J-G. Zhang, Z-G. Lei, J-J. Zhu and F-Y. Xing, Isobaric Vapor - Liquid Equilibrium for Ethyl Acetate + Ethanol + 1-Ethyl-3-methylimidazolium Tetrafluoroborate. J. Chem. Eng. Data. 200954 (2) 193-197.

[13] D-S. Deng ,Y-Z. Qiao, D-X. Ji, Y. Ge, L-Z. Zhang, Measurement and modeling of vapor-liquid equilibrium for ternary system water +2 -propanol +1-Butyl-3-methylimidazolium chloride. Chin. J. Chem. Eng. 22 (2014) 164-169.

[14] D-S. Deng, R-F. Wang, L-Z. Zhang, Y. Ge, J-B. Ji, Vapor-liquid equilibrium measurements and modelling for the ternary system (water +2 -propanol +1-butyl-3-methylimidazolium acetate). Phys. Chem. Liq. 50 (2012) 504-512.

[15] L-Zh. Zhang, J-Zh. Han, D-S. Deng, J-B. Ji, Selection of ionic liquids as entrainers for separation of water and 2-propanol. Fluid Phase Equilib. 255 (2007) 179-185.

[16] Z.-G. Zhang, D.-H. Huang, W.-X. Li, Entrainer selection for separating tetrahydrofuran/water azeotropic mixture by extractive distillation, Sep. Purif. Technol. 122 (2014) 73-77.

[17] Wenxiu Li, Dezhang Sun, Tao Zhang, Shangwu Dai, Fenjin Pan, Zhigang Zhang*, Separation of acetone and methanol azeotropic system using ionic liquid as entrainer, Fluid Phase Equilib. 383 (2014) $182-187$.

[18] C. L. Yaws, H. C. Yang, To estimate Vapor pressure easily Hydrocarbon Processing, 1989, P65.

[19] H. Renon, J. M. Prausnitz, Local composition in thermodynamic excess functions for liquid mixtures. AIChE J. 14 (1968) 135-144.

[20] P. Marzal, J. B. MontÓn, M.A. Rodrigo, Isobaric vapor-liquid equilibria of the water + 2-propanol system at 30 , 60 ,and $100 \mathrm{kPa}$. J. Chem. Eng. Data 41 (1996) 608-611.

[21] J. Li, X. Yang, K. Chen, Sifting Ionic Liquids as Additives for Separation of Acetonitrile and Water 
Azeotropic Mixture Using the COSMO-RS Method. Ind. Eng. Chem. Res. 51 (2012) 9376-9385.

[22] Mercedes Lo 'pez-Pastor, Marı'a Jose' Ayora-Canada, Miguel Valca 'rcel, and Bernhard Lendl, Association of Methanol and Water in Ionic Liquids Elucidated by Infrared Spectroscopy Using Two-Dimensional Correlation and Multivariate Curve Resolution. J. Phys. Chem. B 110 (2006) 10896-10902.

[23] L C Cammarata, S G Kazarian, P A Salter, T Welton. Molecular states of water in room temperature ionic liquids. Phys. Chem. Chem. Phys. 3 (2001) 5192-5200.

[24] J. Dhanalakshmi, P. S. T. Sai, and A. R. Balakrishnan Study of Ionic Liquids as Entrainers for the Separation of Methyl Acetate-Methanol and Ethyl Acetate - Ethanol Systems Using the COSMO-RS Model. Ind. Eng. Chem. Res. 52 (2013) 16396-16405.

[25] Catarina M. S. S. Neves, Marta L. S. Batista, Ana Filipa M. Cla'udio, Lu1́s M. N. B. F. Santos, Isabel M. Marrucho, Mara G. Freire, and Joa o A. P. Coutinho, Thermophysical properties and water saturation of $\left[\mathrm{PF}_{6}\right]^{\top}$ based ionic liquids. J. Chem. Eng. Data 55 (2010) 5065-5073. 